\title{
The role of the voluntary sector: pump primer or pit prop?
}

\author{
Mary Baker
}

\begin{abstract}
The role of the voluntary sector is discussed from the standpoint of the Parkinson's Disease Society (PDS). The Society's Welfare Service is committed to listening to the needs of people with PD and their carers. One example of the "pump-priming" process is the Neurocare Project. Models of good practice should benefit increasing numbers of patients and carers throughout the country. Another form of "pump-priming" is the developing cooperation of charities, including the PDS, in "Neuro-Concern". However, charities must expect to be "pit props" to some extent. They should be valued partners with others in the planning of improved services for people with neurological conditions.
\end{abstract}

I would like to outline the role which a voluntary organisation can play in helping people with Parkinson's disease. Knowledge of the medical and scientific aspects of Parkinson's disease is very important and it is encouraging to learn from seminars and professional journals about the advances in understanding which are taking place. I have less confidence that there has been equivalent progress in understanding the personal, social and emotional aspects of this condition.

Parkinson's disease is known to affect people of both sexes and of varying age, social background, nationality and culture. It should be only a small step from this fact to an acknowledgement of the need to understand, in each case, the implications of living with Parkinson's disease. This is best achieved by listening and talking to the experts-those who have the illness and their carers. The Parkinson's Disease Society which was started by patients, relatives and friends has an honourable pedigree in this respect and the early anecdotal information became more clear and reliable when the Society commissioned and published a study of its own members with Parkinson's disease. ${ }^{1}$ Since then a variety of special projects, working parties and seminars have added to our recognition of the value of seeing things through the eyes of people with Parkinson's disease and their carers.

We view Parkinson's disease as a disorder of movement which affects learned voluntary actions. Such a definition directs attention to the very wide range of activities which can be affected-from basic tasks, such as walking, talking, toileting and dressing to social, value- laden actions like writing, driving, dancing and making love. Parkinson's disease can affect every aspect of life but because it is also variable in its effect, no two people's experience of it will be quite the same. Another source of variation in impact is the family situation. A patient may already be caring for another family member or have special responsibilities or anxieties which will influence his or her ability to cope with the diagnosis or respond fully to treatment.

For the person with Parkinson's disease, common problems include loss of mobility, difficulties in communicating and problems with personal care, all of which can lead to isolation, boredom, frustration or depression and to financial difficulties and traumatic questions of role reversal. There is likely to be grief at the loss of independence and self-esteem and feelings of embarrassment and loss of dignity. Problems with communication (which in Parkinson's disease can affect almost every channel from facial expression and speech to body language and writing) can result in withdrawal from social activity, loss of companionship and the breakdown of relationships. Dr Pentland and his colleagues ${ }^{2}$ have illustrated the effect of reduced expression on professionals and we need to remind ourselves of the likely effect on those who are constantly denied the reward in smiles and indications of interest which all our cultural conditioning leads us to expect.

For the carer, the common problems include lack of freedom, loss of personal leisure interests and the loss of a sense of identity plus tiredness due to constant demands. Certain features of Parkinson's disease and its treatment such as unpredictable "freezing", the on/ off syndrome and abnormal involuntary movements require very special qualities of patience, tolerance and understanding from carers not just for a week or a month but for years and years.

Voluntary organisations have a long tradition of providing broad support to those searching for that elusive "wealth", the cure or improved treatment of conditions such as Parkinson's disease so in that sense it is certainly appropriate for them to act as "pit props". It may be worth considering whether that metaphor implies considerable input from other sources and whether the balance of contributions has changed unhelpfully in recent years. The Parkinson's Disease Society and other neurological charities continue to play an important role in supporting medical research.

On the welfare side, we have been more involved in "pump priming", that is, in finding 
and encouraging new models of care or management which take account of the messages received from people with Parkinson's disease and their families. So we have listened, and responded with special projects or working parties, to the expressed needs for better communication of the initial diagnosis, for more information at all stages, for early access to counsellors, therapists and dietitians, for greater understanding of patients in hospital, for more support for carers, for appropriate and accessible driving assessment, and for better communication with GPs. Most of our welfare projects seek to address these needs by providing initial funds to allow models of good practice to be tried and evaluated. There is no intention in any project of providing permanent funding and further details about one particular project will help to clarify this issue and illustrate our willingness to cooperate with both voluntary and statutory agencies.

In 1985 the Parkinson's Disease Society's Welfare Advisory Panel and Council approved a project based in Romford, Essex, which specifically aimed to create a long term strategy of care and management based on the needs identified by patients and their families. The objectives of the project are summarised in a new information leaflet ${ }^{3}$ as follows;

“The Parkinson's pilot project attempted to create a new model for the relationships between patients and professionals and between hospital and community services. Its main features were:

a) A multi-disciplinary team which straddles the hospital-community divide and in which any member can suggest initiatives;

b) Recognition of patients' needs for information (adapted to their own situation) at all stages of their illness but especially around the time of diagnosis;

c) Special attention to the patient as a "whole person" and to the social and emotional aspects of long term illness;

d) The involvement of patients and carers in monitoring and decision making".

The results of the pilot study were published in $1988^{4}$ and some preliminary comparative data were presented at the Society for Research in Rehabilitation's Summer conference in 1989. Meanwhile a great deal of time and effort has been devoted to extending the project's overall principles to other neurological conditions and so improving the chances of both short term funding and long term integration into the statutory services. The Neuro-care Team (of consultant neurologist and associated doctors, full time coordinator/counsellor, three part time therapists, part time dietitian and part time secretary) now offer a service to those that have been newly diagnosed with motor neuron disease, multiple sclerosis, dystonia and ataxia as well as Parkinson's disease. It is funded for three years from April 1990 by Joint Funds from the Health Authority and by contributions from all the participating voluntary organisations. The challenge is not only to try to make the team a permanent feature of the Health and Social Services in Romford but to promote this approach to care throughout the country.

Travelling around the country I find great support for this approach from patients and families and evidence of considerable avoidable suffering where the "Romford" principles of care are not in evidence.

The cooperation with other neurological charities achieved in the Romford Neuro-care Project is now being actively pursued at national level in the creation of an organisation called "Neuro-Concern". The smaller and medium size neurological charities which have come together in "Neuro-Concern" believe that they have a major contribution to make in the planning and delivery of services for people with chronic neuro-degenerative conditions and the group is already working with neurologists and with planners and administrators in the Health and Social Services. The aim is to ensure that effective provision is made for people with neurological conditions as the changes in the Health and Social Services come into effect over the next few years. The concluding paragraph of the Neuro-care leaflet could apply equally well to "Neuro-Concern";

"The members of the Neuro-care Team do not claim to know all the answers to the questions posed by these complex conditions. They only claim to have tried to listen to what patients and their families say and to be ready to learn from each new experience. The team will not always be able to provide exactly what people want but will work with them to explore the range of available solutions to each individual problem. Overall the objective is to help people with progressive neurological conditions have the best possible quality of life".

In conclusion, my answer to the question at the beginning is to accept the validity of both "pit prop" and "pump primer" roles but to argue strongly for another one-that of valued partner in the battle to improve services for people who are our members and your patients. One part of that partnership will be to allow us to participate in the planning of services and to place our special knowledge of the needs and wishes of people with neurological conditions in the hands of those, like yourselves, who can change things for the better.

\footnotetext{
1 Oxtoby M. Parkinson's disease patients and their social needs. London: Parkinson's Disease Society, 1982.

2 Pentland B, Pitcairn TK, Gray JM, Riddle WJR. The effects of reduced expression in Parkinson's Disease on impression formation by health professionals. Clinical Rehabilitation 1987;1:307-13.

3 Neuro-care Romford. Leaflet from the Neuro-care Office, Harold Wood Hospital, Romford, Essex, RM3 0BE, UK. 4 Oxtoby M, Findley L, Kelson N, et al. A strategy for the management of Parkinson's disease and for the long term support of patients and their carers. London: Parkinson's Disease Society, 1988.
} 